\title{
Rapid Differentiation of Bacterial Meningitides by Direct
}

\section{Gas-Liquid Chromatography}

\author{
Haragopal Thadepalli, Pijush K. Gangopadhyay, Ali Ansari, \\ Gary D. Overturf, Vinod K. Dhawan, and Ashis K. Mandal, Division of \\ Infectious Diseases, Departments of Medicine, Pathology and Surgery, \\ Charles R. Drew Postgraduate Medical School, University of California at \\ Los Angeles Schools of Medicine, Los Angeles, California 90059
}

A B S T R A C T Rapid identification of Haemophilus influenzae and other bacillary meningitides was attempted by gas-liquid chromatography (GLC) of the metabolic by-products in broth cultures and in cerebrospinal fluid (CSF) samples obtained from experimental meningitis produced in New Zealand White male rabbits. These results were correlated with the GLC of CSF of meningitis patients. A major peak with retention time of succinic acid was found in the broth cultures of all bacilli tested including $H$. influenzae, Escherichia coli, Enterobacter cloacae, Klebsiella pneumoniae, Proteus mirabilis, Citrobacter freundii, Pseudomonas aeruginosa, and Listeria monocytogenes. Succinic acid was also found in the CSF of experimental meningitis and in the CSF of all patients with $H$. influenzae and Esch. coli meningitis. This peak was not detected in the blood samples of experimental animals. It was also absent in the broth cultures of all of the gram-positive and gram-negative cocci tested, such as Streptococcus pneumoniae and Neisseria meningitidis. Succinic acid, which appears to be a by product of fermentation, persisted as a clear cut marker in $H$. influenzae meningitis for at least $3 \mathrm{~d}$ after the initiation of treatment. In one patient, the succinic acid peak disappeared during treatment and reappeared with a clinical relapse. Clearly, the presence of succinic acid that can be rapidly detected by GLC in the CSF excludes pneumococcal or meningococcal meningitis and strongly suggests $H$. influenzae or other bacillary meningitides.

\section{INTRODUCTION}

Cerebrospinal fluid (CSF $)^{1}$ pleocytosis, glucose, and protein levels are nonspecific and may be misleading

Received for publication 15 October 1979 and in revised form 14 December 1981.

${ }^{1}$ Abbreviations used in this paper: CSF, cerebral spinal fluid; GLC, gas-liquid chromatography. in diagnosis of purulent meningitis; about $12 \%$ of CSF by Gram's stain and $20 \%$ by culture are falsely negative $(1,2)$. Lactic acid levels in CSF are increased in cases of purulent bacterial meningitis (3-5). Leukocyte lactate dehydrogenase activity $(6,7)$ and lysozyme levels increase in bacterial, but not in viral meningitis (8-10). Other diagnostic markers of purulent meningitis include: decreased CSF cyclic adenosine $3^{\prime}, 5^{\prime}$ monophosphate (11); increased lysozyme activity (10); increased creatinine phosphokinase (12); increased Creactive protein levels (13); and increased IgM concentration in the CSF (14). But none of these tests is specific for bacillary meningitis. Limulus lysate test can help to quickly differentiate gram-negative from gram-positive meningitis $(1,15-17)$, but it does not differentiate neisserial from gram-negative bacillary meningitides. Counterimmunoelectrophoresis of CSF is specific, but false negative results have been reported in 10 to $23 \%$ of patients (18-21). Gas-liquid chromatography (GLC) was found to be useful for rapid identification of anaerobic bacteria (22) and fungi (23). It is also used for rapid diagnosis of gram-negative anaerobic bacterial infections $(24,25)$. Gram-negative meningitis, other than $H$. influenzae is on the increase and is attended with $44 \%$ mortality (26). By the age of 10 , nearly 1 of every 1,500 children are still affected by $H$. influenzae meningitis and it is fatal in $5.7 \%$ (27). Markers were sought for rapid diagnosis of gram-negative bacillary meningitis by GLC. Rapid differentiation of pneumococcal and meningococcal meningitis from that of $\boldsymbol{H}$. influenzae and gram-negative bacillary meningitides is of obvious therapeutic importance.

\section{METHODS}

The bacteria used in this study were obtained from CSF of meningitis patients. All isolates were reidentified before doing GLC. The bacteria tested were Streptococcus pneumoniae, Neisseria meningitides, Haemophilus infuenzae, 
Escherichia coli, Enterobacter cloacae, Klebsiella pneumoniae, Proteus mirabilis, Citrobacter freundii, Listeria monocytogenes, and Pseudomonas aeruginosa.

Experimental meningitis. Bacterial meningitis was produced in male New Zealand White rabbits weighing 2-2.5 kg. each, by direct injection of overnight growth of bacterial broth cultures into the cisterna magna. Briefly, the procedure was as follows: under ether anesthesia, the atlanto-occipital joint was surgically exposed and injected with $0.2 \mathrm{ml}$ of overnight culture of bacteria washed and resuspended with saline to contain $10^{7}$ to $10^{8} / \mathrm{ml}$ of viable cells, into the cisterna magna with a 26-gauge needle. The incision was then closed with interrupted nylon stitches. 8 rabbits were inoculated with meningococcus; 10 with pneumococcus; 5 with Esch. coli, and 18 others with $H$. influenzae. Because, the 18 adult rabbits failed to develop meningitis with $H$. influenzae, it was repeated in eight additional newborn rabbits, 6 to 10 $\mathrm{d}$ old and all eight developed meningitis. For negative control, six adult and two infant rabbits were operated but not inoculated with bacteria. In cases of death, the CSF was withdrawn from the cisterna magna and an autopsy was performed. All surviving rabbits were killed on the 3rd d after the CSF samples were collected. These CSF samples were cultured and studied by GLC methods.

Clinical specimens. 149 CSF samples were collected by lumbar puncture from 81 patients, 66 of whom had proven meningitis, and 15 had no evidence of meningitis. The specimens were sorted into six separate groups: $(a)$ Untreated meningitis: this included 49 patients with bacterial meningitis other than tuberculosis, as proved by positive CSF cultures. 18 patients had pneumococcal meningitis; 16 had $H$. influenzae; 14 had $N$. meningitidis and one had Esch. coli meningitis. (b) Partially treated meningitis: Specimens of CSF were obtained from patients in group (a) following 1$3 \mathrm{~d}$ of antibiotic treatment. In 19, specimens were obtained 1-3 d after treatment, and in 32 , specimens were obtained after 4-7 d of treatment. (c) Fully treated bacterial meningitis: In 16, CSF samples were obtained after antibiotic treatment, usually $>7 \mathrm{~d}$. $(d)$ Aseptic meningitis: Nine patients had aseptic meningitis. They had sterile CSF cultures and $100-1,000$ leukocytes $/ \mathrm{ml}^{3}$ in the pretherapy CSF samples. $(e)$ Tuberculosis and fungus meningitis: Two patients had tuberculosis meningitis and six had fungal meningitis, including four Cryptococcus neoformans and two Coccidioides immites meningitides. $(f)$ Controls: 15 patients had lumbar punctures for noninfectious causes, such as delirium tremens and cerebrovascular accidents; their spinal fluid samples were sterile and had normal cell counts, glucose, and protein levels. These were included as controls.

Fermentation by-products of spent-broth cultures. A single colony of culture from each agar plate was transferred to $4 \mathrm{ml}$ of brain heart infusion broth (California Laboratory Industries, North Hollywood, CA) and incubated at $37^{\circ} \mathrm{C}$ for $18 \mathrm{~h}$. A $0.5-\mathrm{ml}$ portion of this broth culture was mixed with $0.2 \mathrm{ml}$ of $50 \% \mathrm{H}_{2} \mathrm{SO}_{4}$ and $1.0 \mathrm{ml}$ of methanol, heated at $60^{\circ} \mathrm{C}$ for $30 \mathrm{~min}$, cooled, diluted with $1 \mathrm{ml}$ of water, equilibrated with $0.5 \mathrm{ml}$ of chloroform, centrifuged, the bottom organic layer separated, and a $2-\mu l$ portion of this layer was used for GLC. For control, a random sample of brain heart infusion agar was also chromatographed. Benzoic acid was used as an internal standard to quantitate lactic acid levels.

Analysis of CSF. The CSF samples were also processed and chromatographed similar to methods described above. The CSF samples from both experimental and clinical meningitis were also processed in the same manner with one difference, i.e., $0.2 \mathrm{ml}$ of CSF from rabbits and $0.5 \mathrm{ml}$ of clinical specimens were used for GLC studies. Most specimens were processed on the same day, but none were left in the refrigerator for $>48 \mathrm{~h}$.

GLC. A stainless steel column with $3 / 8$ in. o.d. containing $15 \%$ diethylene glycol succinate on 80-100 mesh chromosorb $\mathrm{W}$-AW was used for organic acid analysis of broth cultures and cerebrospinal fluid samples. The column was fitted to Varian Aerograph (Varian Associates, Inc., Sunnyvale, CA) model 2400 gas chromatograph. Broth cultures and the CSF specimens were assayed on this column, programmed from $90^{\circ}$ to $140^{\circ} \mathrm{C}$ at the rate of $6^{\circ} \mathrm{C} / \mathrm{min}$ and subsequently maintained at $140^{\circ} \mathrm{C}$ for $10 \mathrm{~min}$. The gas chromatograph was equipped with flame ionization detector; the flame was maintained by a mixture of hydrogen and air with flow rate of $30 \mathrm{ml}$ and $250 \mathrm{ml} / \mathrm{min}$, respectively. The carrier gas was chromatographic grade nitrogen (Big Three Industries, Long Beach, CA) at a flow rate of $40 \mathrm{ml} / \mathrm{min}$. A Hewlett-Packard reporting integrator, model 3380A (Hewlett-Packard Co., Palo Alto, CA), was used for quantitation.

\section{RESULTS}

\section{Fermentation by-products in spent-broth cultures (Fig. 1)}

Lactic acid. The lactic acid levels were elevated in the broth cultures of all bacterial isolates tested, and they were $25.2 \pm 9.6 \mathrm{mg} / \mathrm{dl}$ of broth. Pneumococcus produced the highest quantity of lactic acid $(136.8 \pm 21.6 \mathrm{mg} / \mathrm{dl})$, followed by $N$. meningitidis $(87.6 \pm 18.0 \mathrm{mg} / \mathrm{dl})$ and $H$. influenzae $(66.0 \pm 14.4$ $\mathrm{mg} / \mathrm{dl})$.

Succinic acid. No succinic acid was detected in the broth cultures of any of the gram-positive or gramnegative cocci tested such as Strep. pneumoniae and $N$. meningitidis. On the other hand, succinic acid was present in the broth cultures of all bacilli tested including $H$. influenzae, Esch. coli, Ent. cloacae, $K$. pneumoniae, Prot. mirabilis, C. freundii and Pseud. aeruginosa. A gram-positive bacillus, L. monocytogenes also showed the succinic acid peak. The succinic acid peak was taller than that of lactic acid with all gram-negative bacilli except Pseud. aeruginosa. The succinic acid to lactic acid ratio was $<1$ with all gramnegative bacilli tested except Pseud. aeruginosa.

\section{Experimental meningitis in rabbits (Fig. 2)}

The CSF obtained from all rabbits that were operated but not inoculated with bacteria showed no lactic acid or succinic acid peak. High lactic acid levels were found in the CSF of all inoculated rabbits that developed meningitis. Inoculation of $H$. influenzae, failed to cause meningitis in all adult rabbits whereas it caused meningitis in all young rabbits. The adults appeared resistant to this infection. The succinic acid levels were high in the CSF of all infant rabbits that developed $H$. influenzae meningitis. Succinic acid was absent in all 18 adult rabbits that failed to develop $H$. 

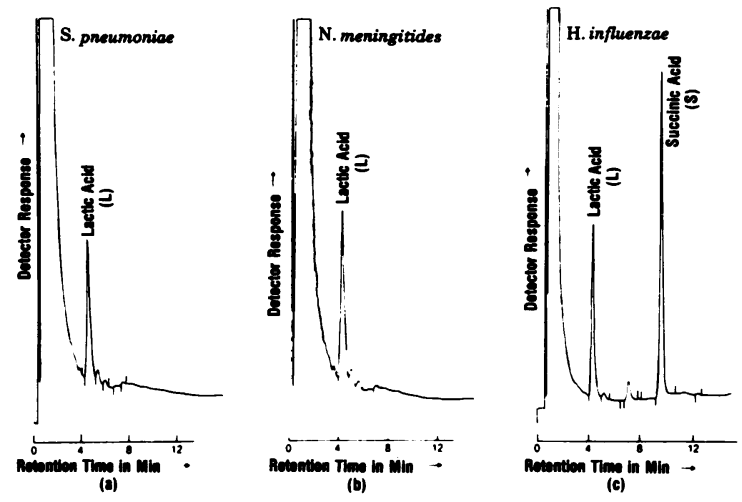

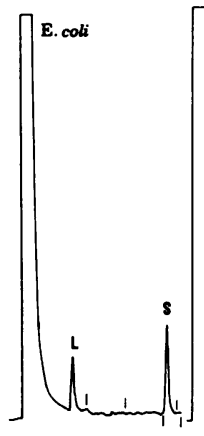

(d)

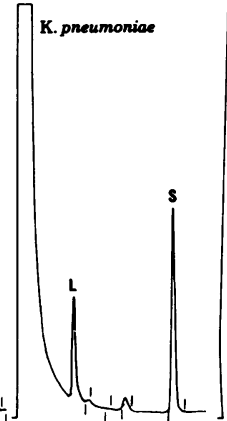

(a)

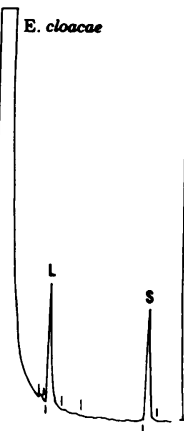

(f)

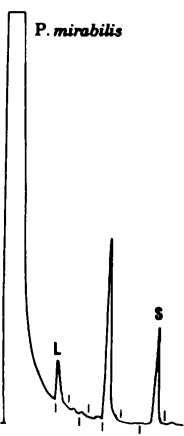

(g)

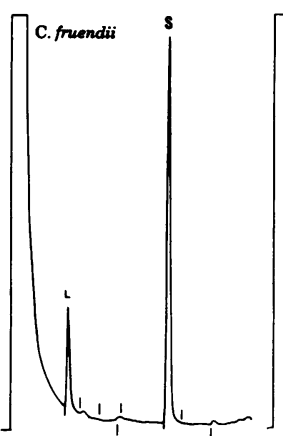

(h)

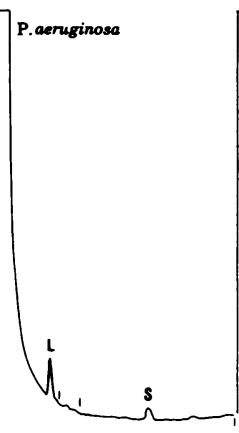

(i)

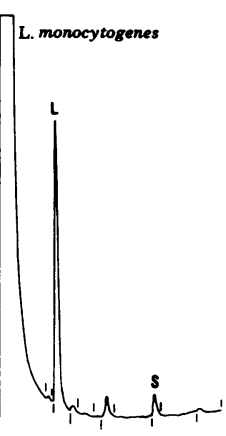

(j)
Figure 1 Spent-broth cultures: (a) pneumococcus, (b) meningococcus, and (c) $H$. influenzae. Note the absence of succinic acid peak (S) with pneumococcus (a), meningococcus (b), and the presence of it with $H$. influenzae (c). GLC patterns of spent-broth cultures of bacilli: (d) E. coli, (e) $K$. pneumoniae, (f) Ent. cloacae, (g) Prot. mirabilis, (h) C. freundii, (i) Pseud. aeruginosa, and (j) L. monocytogenes. Succinic acid peak was present in all. The succinic peak was larger than lactic acid in all except Pseud. aeruginosa and L. monocytogenes.

influenzae meningitis. The CSF of all five animals infected with Esch. coli also had high succinic acid levels. None of the blood samples drawn from any of these animals had succinic acid. $H$. influenzae meningitis could be readily differentiated from pneumococcal or meningococcal meningitis by the presence of succinic acid. No detectable succinic acid levels were found in the CSF samples of pneumococcal and meningococcal meningitis.

\section{Meningitis in man (Fig. 3)}

Lactic acid. The lactic acid levels in the spinal fluid samples in the controls were $<30 \mathrm{mg} / \mathrm{dl}$ (mean $27.4 \pm 6.8$.), whereas in meningococcal, pneumococcal, and $H$. influenzae meningitis group the levels were clearly $>40 \mathrm{mg} / \mathrm{dl}$. They were $144 \pm 73.2 \mathrm{mg} / \mathrm{dl}$ for pneumococcal, $87.6 \pm 64.5$ for meningococcal, and $64.3 \pm 18.0$ for $H$. influenzae meningitis. The lactic acid levels in all nine patients with aseptic meningitis were slightly above the uninfected controls; $(22.6 \pm 6.1$ $\mathrm{mg} / \mathrm{dl}$ ) but was not diagnostic. The lactic acid levels remained higher than normal for at least $3 \mathrm{~d}$ during treatment and in all cases reverted back to normal after $7 \mathrm{~d}$ of therapy.

Succinic acid. No succinic acid was found in any of the 15 CSF samples of patients without meningitis. It was absent in pneumococcus and meningococcus meningitis and aseptic meningitis. The succinic peak was readily detectable in all cases of $H$. influenzae meningitis and in the patient with Esch. coli meningitis as well. No succinic acid peaks were found in the CSF of two patients with tuberculous meningitis and six with fungal meningitis. The succinic acid peak was noted in the CSF of all 16 patients with $H$. influenzae meningitis. In addition to lactic acid and succinic acid, four other minor unidentified peaks were also found, but they were not considered diagnostic.

During antibiotic therapy, several samples were taken from six patients with $H$. influenzae meningitis (Table I). The succinic acid levels decreased with antibiotic treatment and disappeared before the lactic acid levels reverted back to normal. The succinic acid level persisted for $\sim 72 \mathrm{~h}$ after the beginning of antibiotic treatment. The Gram stain and the cultures became negative before succinic acid altogether disappeared in all patients studied. One patient relapsed after the completion of therapy. In this case, the succinic acid disappeared after $11 \mathrm{~d}$ of therapy and reappeared $12 \mathrm{~d}$ later when the Gram stain and cultures became positive (No. 2 in Table I) again for $H$. influenzae. In this case, the organism was resistant to ampicillin and therefore was treated with chloramphenicol. The CSF samples obtained $6 \mathrm{~d}$ after treatment showed no detectable succinic acid.

Sensitivity of succinic acid levels. The sensitivity of succinic acid levels were determined by performing GLC and total counts of viable cells of $\boldsymbol{E}$. coli and $\boldsymbol{H}$. influenzae at different incubation times i.e., 3, 6, 12, and $24 \mathrm{~h}$ after inoculation of brain heart infusion 


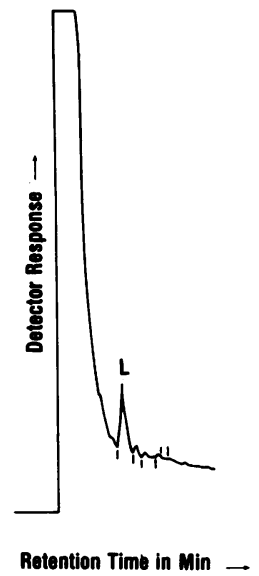

(a)

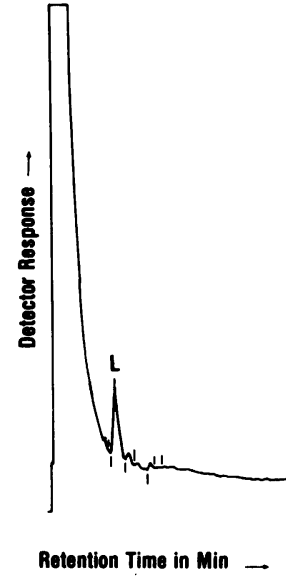

(b)

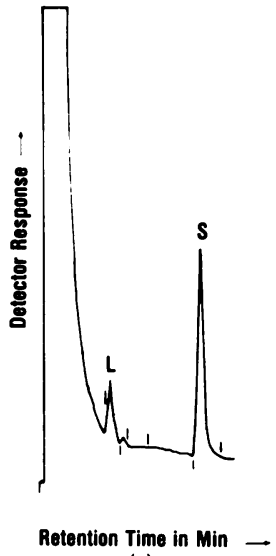

(c)

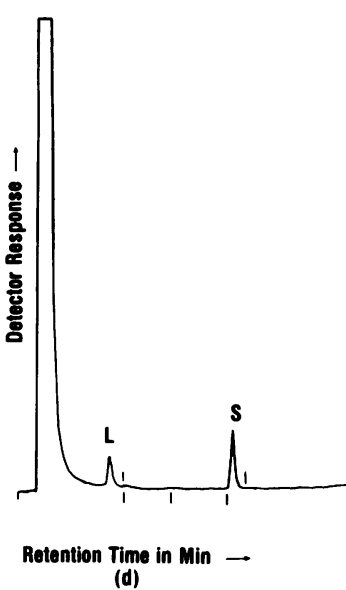

(d)

FIGURE 2 Experimental meningitis in rabbits. Note the presence of succinic acid (S) in the CSF of $H$. influenzae (c) and Esch. coli (d) and the absence of it in meningococcal (a) and pneumococcal (b) meningitis.

broth. Succinic acid was detected at as early as $3 \mathrm{~h}$ after inoculation of broth and at as low a count as 1 $\times 10^{4}$ cells $/ \mathrm{ml}$ of $E$ sch. coli and $H$. infuenzae. Within $3 \mathrm{~h} 1 \times 10^{4} / \mathrm{ml}$ of Esch. coli produced nearly $6 \mathrm{mg} /$ $\mathrm{dl}$ of succinic acid indicating that this method is sensitive enough to detect succinic acid levels in bacillary meningitis.

\section{DISCUSSION}

H. influenzae in children and Esch. coli in the adult are the most common causes of gram-negative meningitis. Nearly $4 \%$ of all cases of meningitis are caused by gram-negative bacilli (26). $69 \%$ of meningitides secondary to neurosurgery and $42 \%$ of meningitides

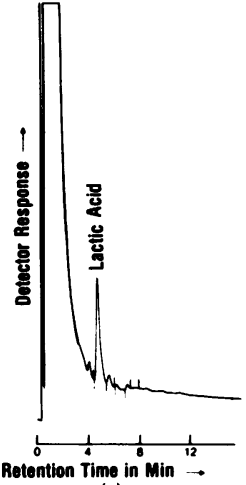

(a)

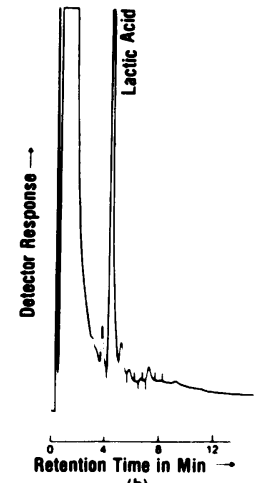

(b)

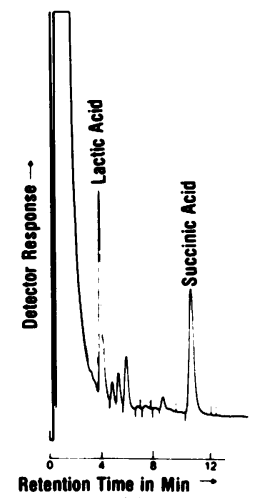

(c)
Figure 3 Meningitis in man. Note the presence of prominent succinic acid peak in the CSF samples obtained from $H$. influenzae meningitis (c). It is characteristically absent in the CSF of patients with meningococcal (a), and pneumococcal (b) meningitis. among the newborn are caused by gram-negative bacilli such as Esch. coli and Klebsiella (28). The need for rapid detection of gram-negative meningitides is therefore obvious.

In our study, the lactic acid levels in the CSF of 81 patients with meningitis were in agreement with that of Controni et al. (4). They were clearly $>40 \mathrm{mg} / \mathrm{dl}$ and remained so for at least $3 \mathrm{~d}$ after therapy.

The rabbit model described by Strausbaugh and Sande (29) was used in this study with one modification; we injected the organism intracisternally without the aid of a stereotactic frame. H. influenzae meningitis was produced by intranasal (30) or intraperitoneal (31) inoculation of the bacteria into infant rats but the older rats are apparently resistant to $H$. influenzae meningitis. The cause for this age-dependent susceptibility is unknown. It is not related to the lack of serum bactericidal activity against $H$. influenzae (32-34). We have also encountered similar age-dependent susceptibility to $H$. influenzae infections in rabbits. Intracisternally injected with $H$. influenzae, all adults failed to develop infection, but all infant rabbits were readily infected.

Direct GLC of CSF obtained in $H$. influenzae and $E s c h$. coli meningitis in rabbits showed succinic acid in quantities greater than lactic acid. Distinctly, succinic acid peak was absent in the CSF samples obtained from experimental pneumococcal and meningococcal meningitis. Succinic acid peak was also found in the CSF samples obtained from all of the 16 patients with $H$. influenzae meningitis. The succinic acid appears to be a by-product of bacterial fermentation. Our in vitro data that large enough quantities of succinic acid can be produced by Esch. coli and $H$. influenzae within $3 \mathrm{~h}$ in broth culture, suggests that it is a sensitive 
TABLE I

Changes in Lactic Acid and Succinic Acid Levels in CSF of $H$. influenzae Meningitis in Man during Antibiotic Therapy Compared with Other Laboratory Data

\begin{tabular}{|c|c|c|c|c|c|c|c|c|}
\hline $\begin{array}{l}\text { Serial no. } \\
\text { and } \\
\text { patient } \\
\text { initials }\end{array}$ & $\begin{array}{l}\text { Date of lumbar } \\
\text { puncture }\end{array}$ & $\begin{array}{l}\text { Gram stain/ } \\
\text { culture result }\end{array}$ & Sugar & Protein & $\begin{array}{c}\text { WBC } \\
\text { per }\end{array}$ & PMN & $\begin{array}{c}\text { Lactic } \\
\text { acid }\end{array}$ & $\begin{array}{c}\text { Succinic } \\
\text { acid }^{\bullet}\end{array}$ \\
\hline & & & $m g / d l$ & $m g / d l$ & $m m^{s}$ & $\%$ & $\mathrm{mg} / \mathrm{dl}$ & \\
\hline \multirow[t]{3}{*}{ I S.H. } & $12 / 19$ & $+/+$ & 35 & 210 & 3,400 & 92 & 56 & 0.40 \\
\hline & $12 / 21$ & $0 / 0$ & 39 & 97 & 300 & 54 & 42 & 0.18 \\
\hline & $12 / 26$ & $0 / 0$ & 54 & 73 & 50 & 9 & 17 & Absent \\
\hline \multirow[t]{3}{*}{2 M.J. } & $12 / 3$ & $+/ 0$ & 10 & 253 & 3,700 & 90 & 78 & 0.52 \\
\hline & $12 / 4$ & $0 / 0$ & 37 & 81 & 180 & 10 & 66 & 0.42 \\
\hline & $12 / 14$ & $0 / 0$ & 57 & 63 & 46 & 0 & 27 & Absent \\
\hline \multirow[t]{3}{*}{ Relapse- } & $12 / 26$ & $+/+$ & 35 & 201 & 1,900 & 27 & 62 & 0.50 \\
\hline & $1 / 2$ & $0 / 0$ & 24 & 47 & 561 & 12 & 65 & Absent \\
\hline & $1 / 6$ & $0 / 0$ & 27 & 47 & 450 & 8 & 60 & Absent \\
\hline \multirow[t]{3}{*}{3 C.M. } & $12 / 6$ & $+1+$ & 30 & 83 & 3,000 & 80 & 108 & 1.01 \\
\hline & $12 / 10$ & $0 / 0$ & 44 & 55 & 389 & 4 & 48 & Absent \\
\hline & $12 / 14$ & $0 / 0$ & 43 & 26 & 50 & 2 & 12 & Absent \\
\hline \multirow[t]{5}{*}{4 D.K. } & $12 / 3$ & $+/+$ & 11 & 141 & 300 & 59 & 74 & 1.10 \\
\hline & $12 / 4$ & $0 / 0$ & 10 & 168 & 3,600 & 66 & 68 & 0.73 \\
\hline & $12 / 6$ & $0 / 0$ & 29 & 96 & & & 44 & Absent \\
\hline & $12 / 13$ & $0 / 0$ & 40 & 71 & & & 22 & Absent \\
\hline & $12 / 24$ & $0 / 0$ & 51 & 66 & & & 8 & Absent \\
\hline \multirow[t]{4}{*}{5 O.R. } & $12 / 10$ & $0 /+$ & 16 & 200 & 2,400 & 76 & 42 & 0.64 \\
\hline & $12 / 12$ & $0 / 0$ & 28 & 119 & 700 & 48 & 40 & 0.26 \\
\hline & $12 / 14$ & $0 / 0$ & 31 & 102 & 588 & 42 & 34 & Absent \\
\hline & $12 / 21$ & $0 / 0$ & 39 & 26 & 144 & $\mathbf{0}$ & 16 & Absent \\
\hline \multirow[t]{4}{*}{6 T.M. } & $12 / 2$ & $+/+$ & 48 & 143 & 13,500 & 90 & 44 & 0.67 \\
\hline & $12 / 3$ & $0 /+$ & 75 & 79 & 720 & 80 & 37 & 0.43 \\
\hline & $12 / 5$ & $0 / 0$ & 60 & 63 & 555 & 76 & 32 & 0.08 \\
\hline & $12 / 14$ & $0 / 0$ & 62 & 38 & 50 & 2 & 16 & Absent \\
\hline
\end{tabular}

- Succinic acid expressed as a ratio of succinic acid to benzoic acid. The higher the ratio, higher the concentration.

+ Gram-negative bacilli seen on Gram stain/positive culture for $\boldsymbol{H}$. influenzae.

PMN, polymorphonuclear leukocytes.

test to detect meningitis when the CSF bacteria counts usually are $>1 \times 10^{3} / \mathrm{ml}$. There seems to be a relationship between succinic acid level and the activity of infection in cases of $\boldsymbol{H}$. influenzae meningitis because it quantitatively decreased during therapy (Table I). In one patient, the succinic acid peak disappeared with clinical improvement and reappeared after $10 \mathrm{~d}$ with a relapse of infection. A large number of cases should be evaluated to assess the value of succinic acid as a marker for gram-negative bacillary meningitis. Succinic acid was also used as a marker for Bacteroides infections $(24,25)$.

Succinic acid may be found in cases of gram-positive bacillary meningitis such as $L$. monocytogenes as it was also found in the broth cultures of this organism Although no succinic acid peak was detected in the CSF of tuberculosis and fungus meningitis, we did not examine them for succinic acid production. Succinic acid is absent in normal spinal fluid which makes it to be a superior marker than lactic acid in the CSF. Clearly, the presence of succinic acid peak in the CSF excludes pneumococcal and meningococcal meningitis and strongly suggests $\boldsymbol{H}$. influenzae or other bacillary meningitides. It is a rapid and sensitive test. It can be done on a routine basis in any laboratory equipped to do gas chromatography. The entire procedure including derivatization and analysis can be done within an hour. 


\section{ACKNOWLEDGEMENT}

We thank Madhis K. Bansal, Ph.D. for providing data on the sensitivity of succinic acid levels.

\section{REFERENCES}

1. Ross, S., W. Rodriguez, G. Controni, G. Korengold, S. Watson and W. Khan. 1975. Limulus lysate test for gram-negative bacterial meningitis. Bedside application. JAMA (J. Am. Med. Assoc.) 233: 1366-1368.

2. Pickens, S., G. Sangster, J. A. Gary, and J. M. Murdock. 1978. The effects of pre-admission antibiotics on the bacteriological diagnosis of pyogenic meningitis. Scand. J. Infect. Dis. 10: 183-185.

3. Bland, R. D., R. C. Lister, and J. P. Ries. 1974. Cerebrospinal fluid lactic acid level and $\mathrm{pH}$ in meningitis. Am. J. Dis. Child. 128: 151-156.

4. Controni, G., W. J. Rodriguez, J. M. Hicks, M. D. Ficke, S. Ross, G. Friedman, and W. Khan. 1977. Cerebrospinal fluid lactic acid levels in meningitis. J. Pediatr. 91: 379384.

5. Brook, I., K. A. Bricknell, G. D. Overturf, and S. M. Finegold. 1978. Measurement of lactic acid in cerebrospinal fluid of patients with infections of the central nervous system. J. Infect. Dis. 137: 384-390.

6. Powers, D. W., and E. M. Ayoub. 1974. Leukocyte lactate dehydrogenase in bacterial meningitis. Pediatrics. 54: 27-33.

7. Feldman, W. E. 1975. Cerebrospinal fluid lactic acid dehydrogenase activity. Am. J. Dis. Child. 129: 77-80.

8. Constantopoulos, A., D. Zoumboulakis, K. Karaboula, and N. Matsaniotis. 1977. Cerebrospinal fluid and serum lysozyme activity in bacterial and viral meningitis. Helv. Paediatr. Acta. 32: 217-220.

9. Klockars, M., S. Reitamo, T. Weber, and Y. Kerttula. 1978. Cerebrospinal fluid lysozyme in bacterial and viral meningitis. Acta Med. Scand. 103: 71-74.

10. Ansari, A., A. Lipsey, and R. Nachum. 1979. Cerebrospinal fluid muramidase levels in meningitis. J. Pediatr. 94: 752-755.

11. Wietzman, S., L. B. Palmer, and S. A. Berger. 1979. Decreased cerebrospinal fluid cyclic adenosine $3^{\prime}, 5^{\prime}$ monophosphate in bacterial meningitis. J. Clin. Microbiol. 9: 351-357.

12. Katz, R. M., and W. Liebman. 1970. Creatinine phosphokinase activity in central nervous system disorders and infections. Am. J. Dis. Child. 120: 543-546.

13. Sabel, K. G., and L. A. Hanson. 1974. The clinical usefulness of C-reactive protein (CRP) determinations in bacterial meningitis and septicemia in infancy. Acta Paediatr. Scand. 63: 381-388.

14. Smith, H., B. Bannister, and M. J. O'Shea. 1973. Cerebrospinal fluid immunoglobulins in meningitis. Lancet. II: 591-593.

15. Nachum, R., A. Lipsey, and S. E. Siegel. 1973. Rapid detection of gram-negative bacterial meningitis by the limulus lysate test. N. Engl. J. Med. 289: 931-934.

16. McCracken, G. H., Jr., and L. D. Sarff. 1976. Endotoxin in cerebrospinal fluid. Detection in neonates with bacterial meningitis. JAMA (J. Am. Med. Assoc.) 235: 617620.

17. Jorgensen, J. H., and J. C. Lee. 1978. Rapid diagnosis of gram-negative bacterial meningitis by the limulus endotoxin assay. J. Clin. Microbiol. 7: 12-17.

18. Coonrod, J. D., and M. W. Rytel. 1972. Determination of aetiology of bacterial meningitis by counterimmunoelectrophoresis Lancet. I: 1154-1157.

19. Feigin, R. D., M. Wong, P. G. Shackelford, B. W. Stechenberg, L. M. Dunkle, and S. Kaplan. 1976. Countercurrent immunoelectrophoresis of urine as well as of CSF and blood for diagnosis of bacterial meningitis. J. Pediatr. 89: 773-774.

20. Colding, H., and I. Lind. 1977. Counterimmunoelectrophoresis in the diagnosis of bacterial meningitis. J. Clin. Microbiol. 5: 405-409.

21. Grandoff, D. M., B. Congeni, R. Baker, Jr., P. Ogra, and G. A. Nankervis. 1977. Countercurrent immunoelectrophoresis in the diagnosis of Haemophilus influenzae type B infection. Am. J. Dis. Child. 131: 1357-1362

22. Holdmen, L. V., and W. E. C. Moore. 1977. Anaerobe Laboratory Manual, VPI Anaerobe Laboratory, Virginia Polytechnic Institute and State University, Blacksburg, Va.

23. Gangopadhyay, P. K., H. Thadepalli, I. Roy, and A. Ansari. 1979. Identification of Candida, Cryptococcus and Torulopsis species by gas-liquid chromatography. J. Infect. Dis. 140: 952-958.

24. Gorbach, S. L., J. W. Mayhew, J. G. Bartlett, H. Thadepalli, and A. B. Onderdonk. 1976. Rapid diagnosis of anaerobic infections by gas-liquid chromatography of clinical specimens. J. Clin. Invest. 57(2): 478-484.

25. Thadepalli, H., and P. K. Gangopadhyay. 1980. Rapid diagnosis of anaerobic empyema by direct gas-liquid chromatography of pleural fluid. Chest. 77: 507-513.

26. Finland, M., and M. W. Barnes. 1977. Acute bacterial meningitis at Boston City Hospital during 12 selected years. J. Infect. Dis. 136: 400-415

27. Goldacre, M. J. 1976. Acute bacterial meningitis in childhood. Lancet. I: 29-31.

28. Mangi, R. J., R. Quintiliani, and V. T. Andriole. 1975. Gram-negative bacillary meningitis. Am. J. Med. 59: 829-836.

29. Strausbaugh, L. J., and M. A. Sande. 1978. Factors influencing the therapy of experimental Proteus mirabilis meningitis in infant rats after intranasal inoculation. $J$. Infect. Dis. 137: 251-260.

30. Moxon, E. R., A. L. Smith, D. R. Averill, Jr., and D. H. Smith. 1974. Haemophilus influenzae meningitis in infant rat after intranasal inoculation. J. Infect. Dis. 129: 154-162.

31. Smith, A. L., D. H. Smith, D. R. Averill, Jr., J. Marino, and E. R. Moxon. 1973. Production of Haemophilus influenzae B meningitis in infant rats by intraperitoneal inoculation. Infect. Immun. 8: 278-290.

32. Shaw, S., A. L. Smith, P. Anderson, and D. H. Smith 1976. The paradox of Haemophilus influenzae type $b$ bacteremia in the presence of serum bactericidal activity. J. Clin. Invest. 58: 1010-1029.

33. Weller, P. F., A. L. Smith, P. Anderson, and D. H. Smith. 1977. The role of encapsulation and host age in the clearance of Haemophilus influenzae bacteremia. J. Infect. Dis. 135: 34-41.

34. Granoff, D. M., and R. Rockwell. 1978. Experimental Haemophilus infuenzae type B meningitis: immunological investigation of the infant rat model. Infect. Immun. 20: 705-713. 